Chronic Obstructive Pulmonary Diseases: Journal of the COPD Foundation

\author{
Original Research
}

\title{
Lung, Fat and Bone: Increased Adiponectin Associates with the Combination of Smoking-Related Lung Disease and Osteoporosis
}

Young Ju Suh, PhD ${ }^{1}$ Merry-Lynn N. McDonald, $\mathrm{PhD}^{2}$ George R. Washko, MD ${ }^{3}$ Brendan J. Carolan, $\mathrm{MD}^{4}$ Russell P. Bowler, MD, PhD ${ }^{4}$ David A. Lynch, $\mathrm{MD}^{4}$ Gregory L. Kinney, MPH, PhD 5 Jessica M. Bon, MD 6 Michael H. Cho, MD, $\mathrm{MPH}^{2}$ James D. Crapo, $\mathrm{MD}^{4}$ Elizabeth A. Regan, MD, $\mathrm{PhD}^{4,5}$ for the COPDGene Investigators

\begin{abstract}
Background: Adiponectin has been proposed as a biomarker of disease severity and progression in chronic obstructive pulmonary disease (COPD) and associated with spirometry-defined COPD and with computed tomography (CT)-measured emphysema. Increased adiponectin plays a role in other diseases including diabetes/ metabolic syndrome, cardiovascular disease and osteoporosis. Previous studies of adiponectin and COPD have not assessed the relationship of adiponectin to airway disease in smokers and have not evaluated the effect of other comorbid diseases on the relationship of adiponectin and lung disease. We postulated that adiponectin levels would associate with both airway disease and emphysema in smokers with and without COPD, and further postulated that body composition and the comorbid diseases of osteoporosis, cardiovascular disease and diabetes might influence adiponectin levels.

Methods: Current and former smokers from the COPD Genetic Epidemiology study (COPDGene) (n= 424) were assigned to 4 groups based on CT lung characteristics and volumetric bone mineral density (vBMD). Emphysema (\% low attenuation area at -950) and airway disease (Wall area \%) were used to assess smoking-related lung disease (SRLD). Group 1) Normal Lung with Normal vBMD; Group 2) Normal Lung and Osteoporosis; Group 3) SRLD with Normal vBMD; Group 4) SRLD with Osteoporosis. Cardiovascular disease (CVD), diabetes, C-reactive protein (CRP) and T-cadherin (soluble receptor for adiponectin) levels were defined for each group. Body composition was derived from chest CT. Multivariable regression assessed effects of emphysema, wall area \%, bone density, comorbid diseases and other key factors on log adiponectin.

Results: Group 4, SRLD with Osteoporosis, had significantly higher adiponectin levels compared to other groups and the effect persisted in adjusted models. Systemic inflammation (by CRP) was associated with SRLD in Groups 3 and 4 but not with osteoporosis alone. In regression models, lower bone density and worse emphysema were associated with higher adiponectin. Airway disease was associated with higher adiponectin levels when T-cadherin was added to the model. Male gender, greater muscle and fat were associated with lower adiponectin.

Conclusions: Adiponectin is increased with both airway disease and emphysema in smokers. Bone density, and fat and muscle composition are all significant factors predicting adiponectin that should be considered when it is used as a biomarker of COPD. Increased adiponectin from chronic inflammation may play a role in the progression of bone loss in COPD and other lung diseases.
\end{abstract}

\footnotetext{
Abbreviations: chronic obstructive pulmonary disease, COPD; computed tomography, CT; COPD Genetic Epidemiology study, COPDGene; volumetric bone mineral density, vBMD; smoking-related lung disease, SRLD; cardiovascular disease, CVD; C-Reactive protein, CRP; tumor necrosis factor alpha, TNRa; body mass index, BMI; wall area percentage (as a percentage of total airway area), WA\%; calcium hydroxyapatite, CaHA; quantitative computed tomography, QCT; least squares, LS; analysis of variance, AVONA; coronary artery disease, CAD; forced expiratory volume in 1 second, FEV $\mathbf{1}$; forced vital capacity, FVC
} 
Funding Support: The project described was supported by Award Number R01HL089897 and Award Number R01HL089856 from the National Heart, Lung, and Blood Institute. It was also supported by 1 R01HL122464. The content is solely the responsibility of the authors and does not necessarily represent the official views of the National Heart, Lung, and Blood Institute or the National Institutes of Health. The COPDGene ${ }^{\circledast}$ project is also supported by the COPD Foundation through contributions made to an Industry Advisory Board comprised of AstraZeneca, Boehringer Ingelheim, Novartis, Pfizer, Siemens, Sunovion, and GlaxoSmithKline.

Dr. Young Ju Suh was supported by an Inha University Research grant (Grant no. 50480). None of the funding sources had a role in data collection, data analysis, interpretation or writing of this manuscript.

Date of Acceptance: December 28, 2017

Citation: Suh YJ, McDonald M-LN, Washko GR, et al; for the COPDGene Investigators. Lung, fat and bone: increased adiponectin with the combination of smoking-related lung disease and osteoporosis. Chronic Obstr Pulm Dis. 2018;5(2):134-143. doi: https://doi. org/10.15326/jcopdf.5.2.2016.0174

1 Department of Biomedical Sciences, College of Medicine, Inha University, Incheon, Republic of Korea

2 Channing Division of Network Medicine, Brigham and Women's Hospital, Harvard Medical School, Boston, Massachusetts

3 Division of Pulmonary and Critical Care Medicine, Brigham and Women's Hospital, Harvard Medical School, Boston, Massachusetts

4 Department of Medicine, National Jewish Health, Denver, Colorado

5 School of Public Health, University of Colorado, Denver

6 University of Pittsburgh, Pittsburgh, Pennsylvania

\section{Address correspondence to:}

Elizabeth A. Regan, MD

1400 Jackson St, K706

Denver, CO 80209

Email: ReganE@NJHealth.org

Phone: 303-398-1531

Fax: 303-270-2249

\section{Keywords:}

adiponectin; osteoporosis; chronic obstructive pulmonary disease; COPD; emphysema; airway disease; smoking-related lung disease; QCT; volumetric BMD; bone mineral density; T cadherin; CDH13; C-reactive protein; systemic inflammation; body composition; muscle area; pectoralis; subcutaneous fat area; visceral fat

\section{Introduction}

Adiponectin is an adipocytokine associated with inflammatory lung diseases, ${ }^{1}$ and has been suggested as a biomarker for chronic obstructive pulmonary disease (COPD). ${ }^{2,3}$ It has homology to tumor necrosis factor alpha (TNF-a) and inhibits the production or action of TNF-a. ${ }^{4}$ Adiponectin is expressed by airway epithelial cells, ${ }^{5}$ secreted into airways via the soluble receptor T-cadherin ${ }^{6}$ and binds to cell surface receptors in the lung. ${ }^{1}$ COPD patients have been reported to have increased adiponectin levels compared to patients with normal lung function ${ }^{7-10}$ and adiponectin has specifically been associated with emphysema, ${ }^{2}$ low body mass index $(\mathrm{BMI})^{7}, \mathrm{COPD}$ progression ${ }^{11}$ and COPD exacerbations. ${ }^{12}$

Biomarkers like adiponectin should relate to specific biologic events -airway inflammation and emphysema, more closely than to reduced spirometry values. In addition, smokers with normal spirometry who don't meet criteria for COPD may still have both emphysema and airway disease with impacts on the biomarker, and smokers who meet spirometric criteria for COPD may have varying amounts of emphysema and airway disease. Biomarker associations may be confounded by the occult presence of comorbid diseases and COPD may present with a variety of comorbid conditions including cachexia, osteoporosis, cardiovascular disease and cancer. ${ }^{13-15}$ Increased adiponectin is also associated with Type 1 diabetes and cardiovascular disease ${ }^{16}$ while Type 2 diabetes and metabolic syndrome are associated with lower adiponectin. Most studies of adiponectin in COPD have not quantified the comorbid conditions that may also affect adiponectin levels.

Adiponectin plays an important role in bone metabolism and osteoporosis pathophysiology, modulating bone formation and remodeling by selectively enhancing stem cell development between adipocyte and bone precursors in the bone marrow in response to reduced energy storage and metabolic needs. ${ }^{17,18}$ Increased levels are associated with reduced: bone mass, lean muscle mass and fat mass. ${ }^{19-22}$ Osteoporosis is common in smoking and COPD patients, present in up to $70 \%$ with severe $\mathrm{COPD},{ }^{23}$ and has also been associated with emphysema severity. $^{24,25}$

Based on those considerations, we hypothesized that the CT characteristics of smoking-related disease (emphysema, airway wall thickening, reduced bone density and body composition measures of fat and muscle) would all influence adiponectin levels. Using a subset of patients from the COPD Genetic Epidemiology 
(COPDGene) biomarkers study we assigned each individual to 1 of 4 groups based on airway disease (wall area percentage), emphysema and bone density.

\section{Methods}

\section{Population}

Participants were 424 non-Hispanic white, current and former smokers with a minimum of 10 pack years smoking who were part of an ancillary biomarker study within the COPD Gene Study. ${ }^{26}$ These individuals were selected from the earlier biomarker study group reported by Carolan et $\mathrm{al}^{2}$ because they also had bone density information available. The relationship of these groups to the main COPDGene study is shown in Figure 1. The major demographic difference between the biomarker group and the main cohort was that it did not contain African American individuals. The biomarkers group also had significantly fewer current smokers than the main cohort. All participants signed informed consent documents and institutional review board approval was obtained at each clinical center.

Participants in the COPDGene study had preand post-bronchodilator spirometry, inspiratory and expiratory CT scans, and provided information about respiratory symptoms, quality of life, medications and comorbid diseases. We identified participants as having cardiovascular disease based on self-reporting of any of the following physician-diagnoses: coronary artery disease (CAD), previous myocardial infarction, angina, congestive heart failure, stroke, transient ischemic attacks or peripheral vascular disease. Diabetes diagnosis was based on self-reporting of physician diagnosis and/or use of diabetes medications. ${ }^{27}$

\section{Study Design}

This was a case control study with a control group and 3 case comparisons. Group 1 was the normal reference control group with no CT airway disease, emphysema

\section{Figure 1. Flow Chart of COPDGene Study and Adiponectin Subgroup}

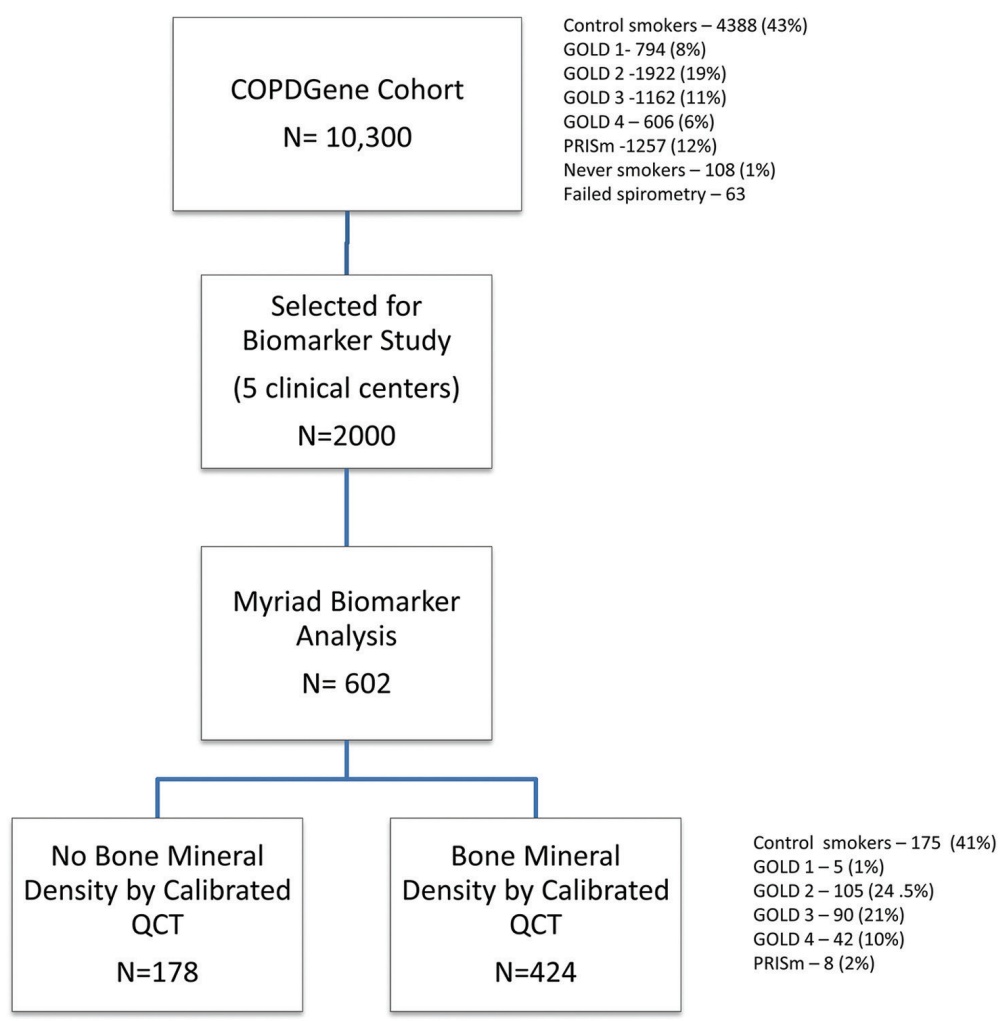

The group of participants studied in this analysis were derived from the COPDGene Phase 1 cohort of 10,300 individuals. The subset of participants who had biomarker analysis done was selected from 5 clinical centers who participated in a specific effort to measure biomarkers and processed blood samples for rapid handling and appropriate storage. There was no effort to make their selection random, but they were not otherwise selected for any characteristic. There were 602 samples that were analyzed for a panel of biomarkers including adiponectin and 446 of those participants had completed their chest CT scan with a calcium calibration phantom that permitted quantitative CT measurement of vertebral cancellous bone density. Distribution of participants by severity of COPD (GOLD stage) is shown for the initial COPDGene cohort and the final study cohort on the right side of the figure.

QCT=quantitative computed tomography; GOLD=Global initiative for chronic Obstructive Pulmonary Disease 
or osteoporosis (Normal Lung-Normal Bone). Group 2 had no CT airway disease or emphysema but had osteoporosis (Normal Lung-Osteoporosis). Group 3 had either CT airway disease or emphysema (smokingrelated lung disease [SRLD])-Normal Bone. Group 4 had either CT airway disease or emphysema (SRLD with Osteoporosis). We further included measures of C-reactive protein (CRP) as a marker of systemic inflammation and T-cadherin as a lung transporter protein for adiponectin in our analysis to define potential pathways and as covariates in models relating adiponectin to lung disease and osteoporosis.

\section{Computed Tomography Scans}

Computed tomography (CT) scans were acquired using multi-detector CT scanners and standardized protocols and quantitative lung analysis as described previously using SLICER software (3D-Slicer, Brigham and Women's Hospital, Boston, Massachusetts) and VIDA analysis (VIDA Diagnostics, Inc., Coralville, Iowa). ${ }^{28}$ Criteria for airway disease was wall area percentage (WA\%) at the segmental level of $>$ (61.2). This value was selected because it was above the 90th percentile for wall area percentage in the COPDGene never-smoker group. Criteria for a participant having abnormal emphysema was: low attenuation area at -950 Hounsfield Units>6.7 (75th percentile for never smoker normal). INTableTM CT (Image Analysis Inc., Columbia, Kentucky) scanner pads containing calcium hydroxyapatite ( $\mathrm{CaHA}$ ) calibration rods were included in the scan field of view to provide calibrated bone density.

\section{Bone Density}

Volumetric bone mineral density (vBMD) over a minimum of 3 vertebral bodies to calculate a mean was measured with automated software, N-VivoTM (image analysis, QSR International, Melbourne, Australia) that calibrated voxel density to the CaHA rods. Criteria for classifying the extent of bone loss were derived using young adult, gender-specific qualitative computed tomography (QCT) reference data from Budoff et al measured with the same software and calibration phantom. ${ }^{29}$ Osteoporosis or low bone density was defined as T-scoreect $<-2.5$ or less using the conventional 2.5 standard deviations below the young adult population mean. Intermediate bone loss was defined as a T-score by Qcr between - 1 and -2.5 and normal bone density was T-score by Qст > -1.0.

\section{Body Composition}

Quantitative assessment of the pectoralis muscle area was performed on a single axial slice of the CT scans just above the aortic arch. The right and left pectoralis major and minor muscles were identified and the area calculated with a final value aggregating the 4 measures. ${ }^{30}$ Lean pectoralis muscle area was calculated by subtracting regions of fat density. Subcutaneous fat area was measured in the chest anterior to the pectoralis muscles. The area measured was bounded by the midline of the sternum and the lateral edge of the pectoralis muscles for each side. The aggregate measure of subcutaneous fat was the sum of those metrics obtained from the left and right anterior chest wall. ${ }^{31}$

\section{Plasma Biomarkers}

Blood samples were obtained from non-fasting participants and collected in a P100 blood collection tube (Becton, Dickenson and Company, Franklin Lakes, New Jersey). Adiponectin, CRP and T-cadherin levels were measured in duplicate using a multiplex biomarker assay (Myriad RBM, Austin, Texas) as previously described. $^{2}$

\section{Statistical Analysis}

We tested the association of the groups to adiponectin levels with and without adjustment for age, gender, BMI and smoking status using multiple regression analysis. Natural log-transformed adiponectin was regressed due to non-normality on residuals. Least squares (LS) means of log adiponectin were estimated in a generalized linear model, after adjusting for age, gender, BMI, and smoking status. For the post hoc test of log adiponectin among the groups, we used the Tukey's method for multiple comparison adjustment.

We looked at factors associated with log adiponectin levels in 2 regression models ( $A$ and $B$ ). The models included age, gender, BMI, current smoking status, pack years, WA\%, emphysema, T-score for BMD, lean pectoralis muscle area, subcutaneous fat area and a history of diabetes or CAD. Model A was the basic model without T-cadherin and Model B included it.

All statistical analyses were performed using SAS (version 9.3, SAS Institute, Cary, North Carolina). Power calculations were done for the 4-group analysis of variance (ANOVA) and for the regression models using PASS (NCSS LLC, Kaysville, Utah). 


\section{Results}

Key demographic and clinical characteristics of the study participants by group are presented in Table 1 . Group 1, Normal Lung-Normal Bone was younger, had fewer pack-years smoking exposure, higher forced expiratory volume in 1 second $\left(\mathrm{FEV}_{1}\right)$, and the lowest adiponectin levels. The mean pectoralis muscle area for this group was higher, as was subcutaneous fat area. There were significant differences in gender and smoking status among the groups ( $p$-value <0.05). Groups 3 and 4, comprising individuals with SRLD, had significantly worse spirometry values consistent with COPD. Subcutaneous fat was significantly lower in both of the osteoporosis groups (Groups 2 and 4). Muscle area was significantly lower in Group 4 with lung and bone disease. BMD, emphysema and WA\% values confirmed the group assignments. CRP was increased significantly in the 2 groups with SRLD $(p<0.001)$, but not in groups 1 and 2 with no lung

\section{Table 1. Population Characteristics by Groups}

\begin{tabular}{|c|c|c|c|c|c|}
\hline & $\begin{array}{c}\text { Group } 1 \\
(\mathrm{~N}=55) \\
\text { Normal Lunga } \\
\text { and } \\
\text { Normal BMD }\end{array}$ & $\begin{array}{c}\text { Group } 2 \\
(\mathrm{~N}=127) \\
\text { Normal Lunga } \\
\text { and } \\
\text { Low BMD }\end{array}$ & $\begin{array}{c}\text { Group } 3 \\
(\mathrm{~N}=52) \\
\text { SRLDa }^{a} \\
\text { and } \\
\text { Normal BMD }\end{array}$ & $\begin{array}{c}\text { Group } 4 \\
(\mathrm{~N}=191) \\
\text { SRLD, } \\
\text { and } \\
\text { Low BMD }\end{array}$ & $P$-value ${ }^{b}$ \\
\hline Age (years) & $58.4(8.3)^{\mathrm{c}}$ & $62.7(8.1)^{\mathrm{d}}$ & $58.9(8.7)$ & $66.2(8.2)^{\mathrm{d}}$ & $<.0001$ \\
\hline Gender (\% Male) & $42 \%$ & $53 \%$ & $33 \%$ & $53 \%$ & 0.0312 \\
\hline Current Smokers (\%) & $24 \%$ & $30 \%$ & $46 \%{ }^{\mathrm{e}}$ & $20 \%$ & 0.0020 \\
\hline Pack Years & $36.1(23.5)$ & $42.7(23.5)$ & $41.5(23.6)$ & $56.1(30.0)^{\mathrm{d}}$ & $<.0001$ \\
\hline BMI $\left(\mathrm{kg} / \mathrm{m}^{2}\right)$ & $29.2(4.7)$ & $27.9(5.0)$ & $29.9(5.6)$ & $27.9(6.0)$ & 0.0099 \\
\hline FEV1 Post- bronchodilator (liters) & $2.8(0.7)$ & $2.9(0.8)$ & $1.8(0.8)^{\mathrm{d}}$ & $1.4(0.8)^{\mathrm{d}}$ & $<.0001$ \\
\hline FEV 1 Percent Predicted & $92.6(15.2)$ & $95.3(17.4)$ & $62.8(24.5)^{\mathrm{d}}$ & $49.7(21.9)^{\mathrm{d}}$ & $<.0001^{\mathrm{f}}$ \\
\hline FEV1/FVC & $0.76(0.06)$ & $0.75(0.09)$ & $0.59(0.18)^{\mathrm{d}}$ & $0.46(0.16)^{d}$ & $<.0001^{\mathrm{f}}$ \\
\hline FVC Percent Predicted & $93.5(10.7)$ & 96.9 (13.3) & $80.7(16.7)^{\mathrm{d}}$ & $80.8(18.8)^{d}$ & $<.0001^{\mathrm{f}}$ \\
\hline BMD (T-Score-QCT) & $-1.7(0.7)$ & $-3.5(0.6)^{d}$ & $-1.8(0.7)$ & $-3.9(0.8)^{\mathrm{d}}$ & $<.0001$ \\
\hline Emphysema & $1.5(1.4)$ & $1.7(1.4)$ & $7.7(12.1)^{\mathrm{d}}$ & $14.6(12.2)^{\mathrm{d}}$ & $<.0001$ \\
\hline Wall Area \% & $58.9(1.6)$ & $58.5(1.7)$ & $63.5(2.1)^{\mathrm{d}}$ & $62.6(3.0)^{\mathrm{d}}$ & $<.0001$ \\
\hline Diabetes & $7 \%$ & $10 \%$ & $25 \%{ }^{\mathrm{e}}$ & $9 \%$ & 0.0095 \\
\hline Cardiovascular Disease (\%)* & $11 \%$ & $17 \%$ & $10 \%$ & $22 \%$ & 0.0833 \\
\hline T-cadherin & $20.2(5.6)$ & $20.7(5.6)$ & $18.5(4.8)$ & $18.4(5.5)^{\mathrm{d}}$ & 0.0007 \\
\hline Adiponectin & $5.0(2.9)$ & $5.8(3.8)$ & $6.1(6.0)$ & $8.4(6.9)^{d}$ & $<.0001$ \\
\hline C Reactive Protein & $3.1(3.2)$ & $3.3(3.5)$ & $5.6(5.2)^{\mathrm{d}}$ & $5.9(9.0)^{\mathrm{d}}$ & $<.0001$ \\
\hline Body Composition & $\mathrm{N}=54$ & $\mathrm{~N}=123$ & $\mathrm{~N}=52$ & $\mathrm{~N}=190$ & \\
\hline Lean Pectoralis Muscle Area & $39.1(14.4)$ & $39.2(11.5)$ & $34.6(10.1)$ & $33.1(10.7)^{\mathrm{d}}$ & $<.0001$ \\
\hline Subcutaneous Fat Area & $67.1(26.5)$ & $54.9(25.1)^{\mathrm{d}}$ & $68.8(27.9)$ & $56.3(29.3)^{d}$ & 0.0004 \\
\hline Abdominal Visceral Fat & $52.3(16.3)$ & $49.2(12.4)$ & $46.1(9.1)$ & $49.7(11.5)$ & 0.244 \\
\hline
\end{tabular}

${ }^{a} \mathrm{COPD}$ classification based on CT imaging characteristics of emphysema (lung attenuation area at $-950 \mathrm{HU}>6.7$ ) and/or bronchial airway thickening (segmental wall area percent $>61.2$ )

${ }^{\mathrm{b}} P$-values obtained by Kruskal-Wallis test for continuous variables, except for FVC percent predicted (by ANOVA), and by $\chi^{2}$ test or Fisher's exact test for categorical variables.

${ }^{\mathrm{c}}$ Means (standard deviations) are shown for continuous variables whereas categorical variables are shown as \% by group.

$\mathrm{d} P$-value $<0.05$ to compare median of each variable between normal (group 1) and patient groups (group 2-4) by the Dunnet's posthoc test for the ranks.

${ }^{e} P$-value $<0.05$ to compare ratio of each variable between normal (group 1) and patient groups (group 2-4) by Fisher's exact test with adjustments by permutation resampling method as a posthoc test.

${ }_{\mathrm{f}}$ Adjusted $p$-value by Bonferroni correction for multiple comparison

The adiponectin level in Group 4 (BOLD) is significantly higher than the reference group (Group 1) and those with bone (Group 2) or lung disease (Group 3) alone. 
disease. Self-reported cardiovascular disease was increased in Group 4 but did not reach statistical significance $(p=0.08)$ while diabetes was significantly higher in Group 3.

We found a significant association of Group 4 with increased adiponectin after adjusting for age, gender, BMI, and smoking status $(p<0.0001)$. In Figure 2 , we illustrate the adjusted means of log adiponectin by group and gender using a generalized linear model, after adjusting for age, BMI, and smoking status. Adjusted means of log adiponectin levels by group tended to be higher in females than those in males so we illustrate a stratified analysis. No significant differences were identified between other groups and power analysis showed $91 \%$ power to detect differences in our model for log adiponectin.

An overall regression model for the whole sample (Table 2, Model A) looked at associations of body composition, WA\%, emphysema and bone density to adiponectin. The model showed that male gender, greater muscle area and subcutaneous fat were associated with lower adiponectin levels. Worse emphysema, and lower bone density were associated with higher adiponectin but WA\% was not significant in this model that did not include T-cadherin. Age, BMI, pack-years smoking, CRP and history of CAD were not significant factors predicting adiponectin levels. A second model (Table 2. Model B) adding T-cadherin showed a higher $\mathrm{R}^{2}$ value for the model, suggesting improved model fit, and wall area percentage became significant $(p=0.028)$.

\section{Discussion}

We found that increased adiponectin levels were associated with the combination of SRLD (based on CT-measured emphysema and airway wall thickening) and osteoporosis in a cohort of smokers, but not with either condition alone. Neither diabetes nor cardiovascular disease was significantly associated

\section{Figure 2. Subtypes of COPD Related to Adiponectin}

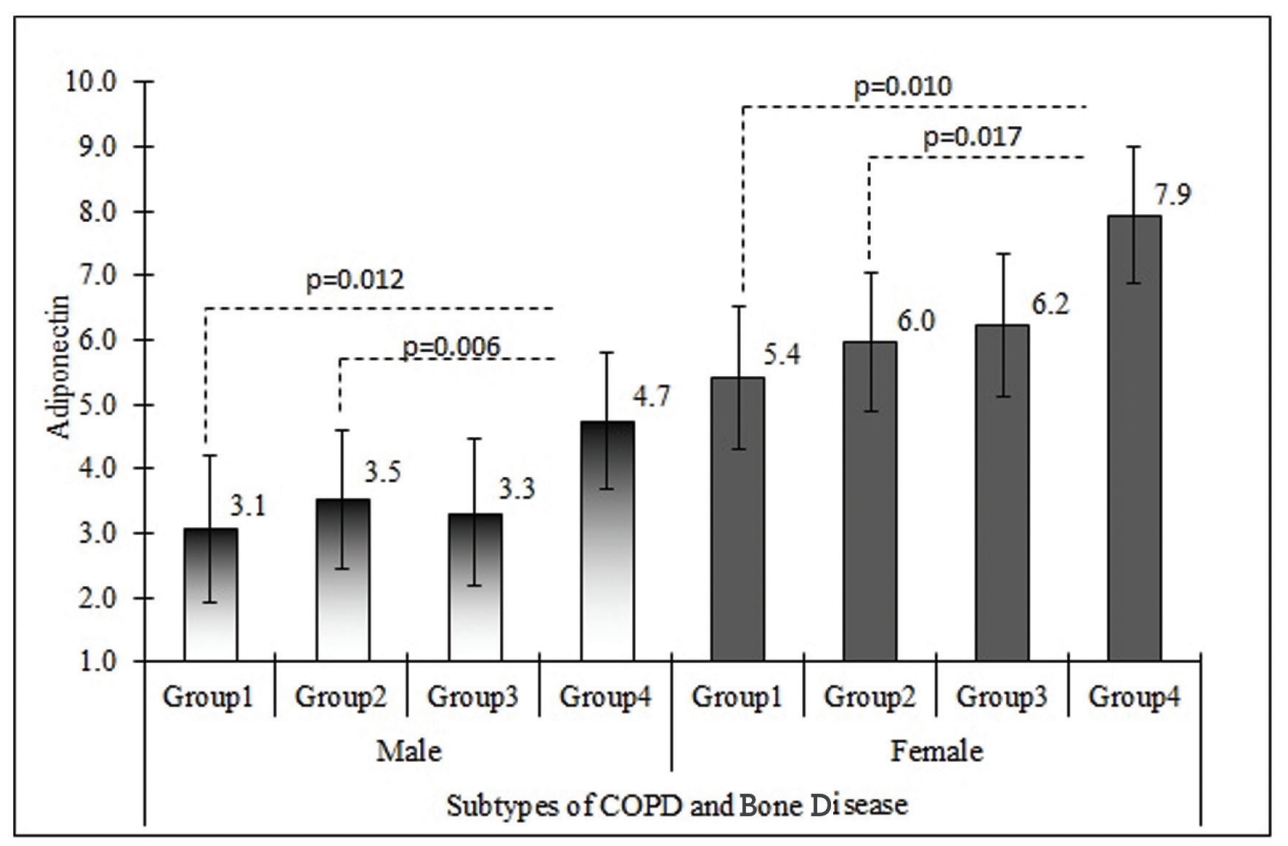

A mean value was calculated for each group and was stratified by gender. Least squares means of $\log$ adiponectin for each of the study groups were estimated in generalized linear models, adjusting for age, BMI, and smoking status by gender.

Study Groups: Group 1 normal lung and bone, Group 2 normal lung and low BMD, Group 3 emphysema and airway disease with normal bone, Group 4 emphysema, airway disease and low BMD.

Women had signficantly higher levels of adiponectin than men, but even after stratification by gender, the group with both COPD and bone loss (Group 4) had significantly higher adiponectin than either COPD or bone loss alone. ( $p$ indicates $P$-value corrected by Tukey's multiple comparison adjustment) with adiponectin in our cohort after adjustment for other variables. Previous studies have focused on associations of adiponectin to either COPD or osteoporosis but have not evaluated the relationship in individuals presenting with both diseases. A history of previous smoking is common in patients over age 45 and COPD may not have been reliably identified or sought in studies of osteoporosis. Likewise, COPD association studies have not quantified bone density in relation to adiponectin and lung disease.

Because COPD is a heterogeneous disease that can involve emphysema and/or airway disease and is diagnosed based on spirometry, not CT, we chose to study the relationship of adiponectin to the measured variables of quantitative emphysema and airway wall thickening as characteristics of SRLD. This allowed us to look 


\section{Table 2. Factors Associated with Adiponectin Levels}

\begin{tabular}{|c|c|c|c|c|}
\hline Variables & $\beta$ & SE & $\mathrm{t}$ & $P$-value \\
\hline \multicolumn{5}{|l|}{ Model A: $\mathbf{R}^{2}=0.38$} \\
\hline Male Gender & -0.590 & 0.082 & -7.15 & $<.0001$ \\
\hline Current Smoking & -0.128 & 0.066 & -1.95 & 0.0521 \\
\hline Volumetric Bone Mineral Density & -0.111 & 0.027 & -4.20 & $<.0001$ \\
\hline Emphysema Percentage & 0.007 & 0.003 & 2.26 & 0.0242 \\
\hline Wall Area Percentage - Segmental Airway & 0.007 & 0.009 & 0.79 & 0.4281 \\
\hline Lean Pectoralis Muscle Area & -0.010 & 0.003 & -2.89 & 0.0040 \\
\hline Subcutaneous Fat Area & -0.008 & 0.001 & -7.05 & $<.0001$ \\
\hline Variables & $\beta$ & SE & $t$ & P-value \\
\hline \multicolumn{5}{|l|}{ Model B (including T-Cadherin): R2 = 0.44} \\
\hline Male Gender & -0.493 & 0.080 & -6.19 & $<0.0001$ \\
\hline Current Smoking & -0.115 & 0.062 & -1.83 & 0.0673 \\
\hline T-cadherin & 0.034 & 0.005 & 6.74 & $<0.0001$ \\
\hline Volumetric Bone Mineral Density & -0.113 & 0.025 & -4.47 & $<0.0001$ \\
\hline Emphysema Percentage & 0.007 & 0.003 & 2.40 & 0.0169 \\
\hline Wall Area Percentage - Segmental Airway & 0.020 & 0.009 & 2.21 & 0.0277 \\
\hline Lean Pectoralis Muscle Area & -0.010 & 0.003 & -3.14 & 0.0018 \\
\hline Subcutaneous Fat Area & -0.006 & 0.001 & -5.63 & $<.0001$ \\
\hline
\end{tabular}

Log-transformed adiponectin was regressed due to non-normality on residuals. Initial model tested: age, gender, BMI, current smoking, pack years, wall area percentage segmental, emphysema, volumetric bone mineral density, T-cadherin, $\mathrm{C}$ reactive protein, history of coronary artery disease, lean pectoralis muscle area and subcutaneous fat area. Stepwise model reduction was performed removing non-significant factors for each model. $\mathrm{BMI}=$ body mass index

smokers when T-cadherin is included as a factor. In a study of the impact of adiponectin on revascularization, the authors found a similar need for T-cadherin to mediate the effect of adiponectin. ${ }^{33}$ Airway disease and emphysema have distinct and additive effects on adiponectin levels.

We find, as others have, that osteoporosis is associated with higher adiponectin levels, but the impact of adiponectin on bone remodeling is complex and distinct from the relationship to lung or heart. Bone mass appears to decrease in response to increased adiponectin levels due to local signals in the bone marrow. ${ }^{18}$ Adipocytes and osteoblasts share a common mesenchymal progenitor

separately at these factors and encompass smokers with and without COPD by spirometry. In our cohort, participants with significant osteoporosis but without lung disease, and those with significant lung disease without osteoporosis did not have increased levels of adiponectin. This suggests that osteoporosis or COPD alone are not associated with elevated adiponectin levels and each may require an additional process to drive up adiponectin levels.

Our findings of elevated adiponectin levels among patients presenting with emphysema, airway disease and osteoporosis are consistent with the underlying biology. Adiponectin receptors are present in the lung, and adiponectin is increased in bronchoalveolar lavage fluid and lung airway epithelial cells of COPD patients. ${ }^{5}$ Lung concentrations of adiponectin are related to T-cadherin transport in normal lung or diffusion from blood in inflammatory lung disease. ${ }^{6,32}$ Airway disease as quantified by WA\% is a significant factor predicting adiponectin levels beyond emphysema in cell in the bone marrow and the regulation of bone formation and fat storage are complexly inter-related. An overall summary of factors related to higher adiponectin is that disease states-more emphysema, thicker airways, lower bone density and reduced muscle mass- are associated with higher adiponectin.

For COPD patients, we hypothesize a pathway where smoking leads to chronic airway inflammation, airway wall thickening, emphysema and tissue wasting; these effects result in increased levels of the antiinflammatory adiponectin modulated, in part, by T-cadherin. As levels of adiponectin rise in response to the inflammatory signal from lung disease, bone loss may be a secondary effect. The demographics of Group 4 with the highest adiponectin levels (older, greater pack years of smoking exposure, generally lower BMI and worse $\mathrm{FEV}_{1}$ ) suggest that the increased adiponectin is occurring in later stage, more severe lung disease. However, longitudinal data will be needed to clarify this. This may explain the increased severity 
of osteoporosis in COPD and the fact that men are affected as frequently as women in this population. ${ }^{34}$

COPD patients are at high risk for both osteoporosis $^{35,36}$ and cardiovascular disease. ${ }^{37} \mathrm{We}$ did not find that self-reporting of CAD was a significant factor predicting adiponectin levels in adjusted models in this study, although a larger proportion of participants reported CAD in Group 4 and more reported diabetes in Group 3. CRP as a marker of systemic inflammation was higher in the 2 groups with COPD, however in adjusted models it was not a significant predictor of adiponectin.

Our study had several limitations. It was not population based and the COPDGene study enhanced recruitment of COPD participants in order to study the disease effectively. Only non-Hispanic white participants were included. Osteoporosis is much less common in African Americans and our findings will need to be evaluated in other racial/ethnic populations. Our ascertainment of comorbid diseases was based on self-reporting of physician diagnoses and medication use. Use of WA\% as a marker of airway disease has not been widely tested against symptoms or disease progression although it reflects an anatomic difference in the airways. It is not part of the Global initiative for chronic Obstructive Lung Disease ${ }^{38}$ criteria for COPD. Analysis of bone density was done using calibrated QCT measures rather than duel-energy $\mathrm{x}$-ray absorptiometry.

Other authors have looked at adiponectin in COPD. Yoon et al looked at patients from the Lung Health Study finding that adiponectin predicted decline in FEV 1 and had a mixed relationship to mortality but did not associate with baseline $\mathrm{FEV}_{1}{ }^{39}$ however they did not assess body composition or bone density. Suzuki compared decline in $\mathrm{FEV}_{1}$ to increased adiponectin and lower leptin/adiponectin ratio in 2 cohorts of COPD patients finding that higher adiponectin and lower ratio were associated with worse outcomes, ${ }^{11}$ but did not report body composition. Their discovery cohort had less than 10\% women and their validation cohort was more balanced but given the significant differences between men and women in adiponectin levels, this may have impacted the generalizability of their results. The study that preceded this analysis from the COPDGene biomarkers cohort by Carolan et al found associations of higher adiponectin to female gender, low BMI, older age, lower FEV 1 and emphysema. ${ }^{2}$ They found an inverse relationship to
2 different airway disease measures (Pi10 and Pi15) but did not have bone density, fat or muscle available for analysis. With this additional body composition data, we can refine the analysis and demonstrate the association of airway disease to adiponectin.

\section{Conclusion}

Smoking-relatedlung diseaseand osteoporosis together are associated with increased adiponectin. T-cadherin, a soluble receptor for adiponectin appears to be a key factor in airway disease that is associated with higher adiponectin levels. Either lung disease or osteoporosis assessed separately without consideration of the other, may lead to erroneous results when using adiponectin as a biomarker of disease. Due to the coexistence of multiple comorbid conditions in the complex diseases of aging, it is important to identify the impact of comorbid conditions on a biomarker and integrate those factors into association studies.

\section{Acknowledgements}

The authors acknowledge the valuable contributions of the COPDGene investigators in developing the cohort and collection of data.

Author Contributions: YJS and EAR contributed to data analysis. RPB contributed to compilation of the biomarker data. DAL, EAR and GRW CT worked on the imaging analysis. YJS, MLM, GRW, BJC, RPB, DAL, GLK, JMB, MHC, JDC, and EAR contributed to the study design, interpretation of data and manuscript preparation. EAR and YJS take overall responsibility for the manuscript.

\section{Declaration of Interest}

$\mathrm{RPB}$ is on the Advisory Board for Boehringer Ingleheim and has received a research grant from MedImmune/Astrazeneca. GRW reports consultancies for Merck, GlaxoSmithKline and Genentech. DAL has received research support from Siemens and reports consultancies for Parexel, Boehringer Ingelheim, Genentech, Gilead Inc, Veracyte Inc, and MEDQIA, Inc. 


\section{References}

1. Ali Assad N, Sood A. Leptin, adiponectin and pulmonary diseases. Biochimie. 2012;94(10):2180-2189.

doi: https://doi.org/10.1016/j.biochi.2012.03.006

2. Carolan BJ, Kim YI, Williams AA, et al. The association of adiponectin with computed tomography phenotypes in chronic obstructive pulmonary disease. Am J Respir Crit Care Med. 2013;188(5)9:561-566.

doi: https://doi.org/10.1164/rccm.201212-22990C

3. Leivo-Korpela S, Lehtimaki L, Vuolteenaho K, et al. Adiponectin is associated with dynamic hyperinflation and a favourable response to inhaled glucocorticoids in patients with COPD. Respir Med. 2014;108:122-128.

doi: https://doi.org/10.1016/j.rmed.2013.08.016

4. Chandran M, Phillips SA, Ciaraldi T, Henry RR. Adiponectin: more than just another fat cell hormone? Diabetes Care. 2003;26(8):2442-2450.

doi: https://doi.org/10.2337/diacare.26.8.2442

5. Miller M, Cho JY, Pham A, Ramsdell J, Broide DH. Adiponectin and functional adiponectin receptor 1 are expressed by airway epithelial cells in chronic obstructive pulmonary disease. $J$ Immunol. 2009; 182:684-691.

doi: https://doi.org/10.4049/jimmunol.182.1.684

6. Hug C, Wang J, Ahmad NS, Bogan JS, Tsao TS, Lodish HF. T-cadherin is a receptor for hexameric and high-molecularweight forms of Acrp30/adiponectin. Proc Natl Acad Sci USA. 2004;101:10308-10313.

doi: https://doi.org/10.1073/pnas.0403382101

7. Tomoda K, Yoshikawa M, Itoh T, et al. Elevated circulating plasma adiponectin in underweight patients with COPD. Chest. 2007;132(1):135-140. doi: https://doi.org/10.1378/chest.07-0227

8. Kirdar S, Serter M, Ceylan E, Sener AG, Kavak T, Karadag F. Adiponectin as a biomarker of systemic inflammatory response in smoker patients with stable and exacerbation phases of chronic obstructive pulmonary disease. Scand J Clin Lab Invest. 2009;69(2):219-224.

doi: https://doi.org/10.1080/00365510802474400

9. Chan KH, Yeung SC, Yao TJ, et al. Elevated plasma adiponectin levels in patients with chronic obstructive pulmonary disease. Int J Tuberc Lung Dis. 2010;14:1193-1200.

10. Breyer MK, Rutten EP, Locantore NW, et al. Dysregulated adipokine metabolism in chronic obstructive pulmonary disease. Eur J Clin Invest. 2012;42(9): 983-991.

doi: https://doi.org/10.1111/j.1365-2362.2012.02686.x

11. Suzuki M, Makita H, Ostling J, et al. Lower leptin/adiponectin ratio and risk of rapid lung function decline in chronic obstructive pulmonary disease. Ann Am Thorac Soc. 2014;11(10):1511-1519. doi: https://doi.org/10.1513/AnnalsATS.201408-351OC
12. Krommidas G, Kostikas K, Papatheodorou G, et al. Plasma leptin and adiponectin in COPD exacerbations: associations with inflammatory biomarkers. Respir Med. 2010;104(1):40-46. doi: https://doi.org/10.1016/j.rmed.2009.08.012

13. Minino AM, Xu J, Kochanek K. Deaths: preliminary Data for 2008. Nat Vital Stat Rep. 2010;59(2):1-52.

14. Lin PJ, Shaya FT, Scharf SM. Economic implications of comorbid conditions among Medicaid beneficiaries with COPD. Respir Med. 2010;104(5):697-704.

doi: https://doi.org/10.1016/j.rmed.2009.11.009

15. Jaramillo JD, Wilson C, Stinson DJ, et al. Reduced bone density and vertebral fractures in smokers: men and COPD patients at increased risk. Ann Am Thorac Soc. 2015;12(5). doi: https://doi.org/10.1513/AnnalsATS.201412-591OC

16. Funahashi T, Nakamura T, Shimomura I, et al. Role of adipocytokines on the pathogenesis of atherosclerosis in visceral obesity. Intern Med. 1999;38(2):202-206. doi: https://doi.org/10.2169/internalmedicine.38.202

17. Biver E, Salliot C, Combescure C, et al. Influence of adipokines and ghrelin on bone mineral density and fracture risk: a systematic review and meta-analysis. Int J Clin Endocrinol Metab. 2011;96(9):2703-2713.

doi: https://doi.org/10.1210/jc.2011-0047

18. Sadie-Van Gijsen H, Crowther NJ, Hough FS, Ferris WF. The interrelationship between bone and fat: from cellular see-saw to endocrine reciprocity. Cell Mol Life Sci. 2013;70(13):2331-2349. doi: https://doi.org/10.1007/s00018-012-1211-2

19. Oshima K, Nampei A, Matsuda M, et al. Adiponectin increases bone mass by suppressing osteoclast and activating osteoblast. Biochem Biophys Res Commun. 2005;331(2):520-526. doi: https://doi.org/10.1016/j.bbrc.2005.03.210

20. Yamaguchi N, Kukita T, Li YJ, et al. Adiponectin inhibits osteoclast formation stimulated by lipopolysaccharide from Actinobacillus actinomycetemcomitans. FEMS Immunol Med Microbiol. 2007;49(1):28-34. doi: https://doi.org/10.1111/j.1574-695X.2006.00164.x

21. Kanazawa I, Yamaguchi T, Yano S, Yamauchi M, Yamamoto M, Sugimoto T. Adiponectin and AMP kinase activator stimulate proliferation, differentiation, and mineralization of osteoblastic MC3T3-E1 cells. BMC Cell Biology. 2007;8:51. doi: https://doi.org/10.1186/1471-2121-8-51

22. Basurto L, Galvan R, Cordova N, et al. Adiponectin is associated with low bone mineral density in elderly men. Eur $J$ Endocrinol. 2009; 160:289-293.

doi: https://doi.org/10.1530/EJE-08-0569

23. Li L, Brennan KJ, Gaughan JP, Ciccolella DE, Kuzma AM, Criner GJ. African Americans and men with severe COPD have a high prevalence of osteoporosis. COPD. 2008;5(5):291-297. doi: https://doi.org/10.1080/15412550802363329 
24. Ohara T, Hirai T, Muro S, et al. Relationship between pulmonary emphysema and osteoporosis assessed by CT in patients with COPD. Chest. 2008;134(6):1244-1249. doi: https://doi.org/10.1378/chest.07-3054

25. Bon J, Fuhrman CR, Weissfeld JL, et al. Radiographic emphysema predicts low bone mineral density in a tobaccoexposed cohort. Am J Respr Crit Care Med. 2011;183(7):885-890. doi: https://doi.org/10.1164/rccm.201004-0666OC

26. Regan EA, Hokanson JE, Murphy JR, et al. Genetic epidemiology of COPD (COPDGene) study design. COPD. 2010;7(1):32-43. doi: https://doi.org/10.3109/15412550903499522

27. Kinney GL, Black-Shinn JL, Wan ES, et al. Pulmonary function reduction in diabetes with and without chronic obstructive pulmonary disease. Diabetes Care. 2014;37(2):389-395. doi: https://doi.org/10.2337/dc13-1435

28. Schroeder JD, McKenzie AS, Zach JA, et al. Relationships between airflow obstruction and quantitative CT measurements of emphysema, air trapping, and airways in subjects with and without chronic obstructive pulmonary disease. AJR Am J Roentgenol. 2013;201(3):W460-470. doi: https://doi.org/10.2214/AJR.12.10102

29. Budoff MJ, Hamirani YS, Gao YL, et al. Measurement of thoracic bone mineral density with quantitative CT. Radiology. 2010;257(2):434-440.

doi: https://doi.org/10.1148/radiol.10100132

30. McDonald ML, Diaz AA, Ross JC, et al. Quantitative computed tomography measures of pectoralis muscle area and disease severity in chronic obstructive pulmonary disease. A crosssectional study. Ann Am Thorac Soc. 2014;11(3):326-334. doi: https://doi.org/10.1513/AnnalsATS.201307-2290C

31. Diaz AA, Zhou L, Young TP, et al. Chest CT measures of muscle and adipose tissue in COPD: gender-based differences in content and in relationships with blood biomarkers. Acad Radiol. 2014;21:1255-261. doi: https://doi.org/10.1016/j.acra.2014.05.013

32. Agusti A, Calverley PM, Celli B, et al. Characterisation of COPD heterogeneity in the ECLIPSE cohort. Respir Res. 2010;11:122. doi: https://doi.org/10.1186/1465-9921-11-122

33. Parker-Duffen JL, Nakamura K, Silver M, et al. T-cadherin is essential for adiponectin-mediated revascularization. J Biol Chem. 2013;288:24886-248897.

doi: https://doi.org/10.1074/jbc.M113.454835

34. Jaramillo JD, Wilson C, Stinson DS, et al. Reduced bone density and vertebral fractures in smokers. men and COPD patients at increased risk. Ann Am Thorac Soc. 2015;12(5):648-656. doi: https://doi.org/10.1513/AnnalsATS.201412-591OC

35. Jorgensen NR, Schwarz P. Osteoporosis in chronic obstructive pulmonary disease patients. Curr Opin Pulm Med. 2008;14(2):122127. doi: https://doi.org/10.1097/MCP.0b013e3282f4efb6
36. Franco CB, Paz-Filho G, Gomes PE, et al. Chronic obstructive pulmonary disease is associated with osteoporosis and low levels of vitamin D. Osteoporos Int. 2009;20(11):1881-1887. doi: https://doi.org/10.1007/s00198-009-0890-5

37. Maclay JD, MacNee W. Cardiovascular disease in COPD: mechanisms. Chest. 2013;143(3):798-807. doi: https://doi.org/10.1378/chest.12-0938

38. Global Initiative for Chronic Obstructive Lung Disease (GOLD). Global strategy for the diagnosis, management and prevention of COPD, 2017. GOLD website. http://goldcopd.org/gold-2017global-strategy-diagnosis-management-prevention-copd/ Published 2017. Accessed March 2018.

39. Yoon HI, Li Y, Man SFP, et al. The complex relationship of serum adiponectin to COPD outcomes COPD and adiponectin. Chest. 2012;142(4):893-899. doi: https://doi.org/10.1378/chest.11-2173 